\title{
The Impact of Different Simulation Modalities on Target Volume Delineation in Breast-Conserving Radiotherapy
}

\author{
Meng Jin (D) ${ }^{1,2, *}$ \\ Xia Liu ${ }^{\prime}, *$ \\ Jiabin $\mathrm{Ma}$ iD $^{\mathrm{I}}$ * \\ Xiansong Sun' \\ Hongnan Zhen' \\ Jing Shen (D) \\ Zhikai Liu id \\ Xin Lian' \\ Zheng Miao' \\ $\mathrm{Ke} \mathrm{Hu}{ }^{\prime}{ }^{\prime}$ \\ Xiaorong Hou (iD) \\ Fuquan Zhang (D) \\ 'Department of Radiation Oncology, \\ Peking Union Medical College Hospital, \\ Chinese Academy of Medical Sciences \\ and Peking Union Medical College, \\ Beijing, People's Republic of China; \\ 2Department of Radiation Oncology, The \\ First Affiliated Hospital of Sun Yat-sen \\ University, Guangzhou, Guangdong, \\ People's Republic of China
}

*These authors contributed equally to this work
Purpose: In the management of breast-conserving radiotherapy, computed tomography (CT) simulation is now commonly used to identify tumor bed while has difficulties defining precisely. We aimed to evaluate the impact of magnetic resonance (MR) and CT simulation on defining the postoperative tumor bed for breast-conserving radiotherapy in patients without the aid of surgical clips.

Methods: From August 2018 to March 2019, twenty patients with $\mathrm{T}_{1-2} \mathrm{~N}_{0} \mathrm{M}_{0}$ breast cancer at our institution were enrolled. All the patients underwent breast-conserving surgery without implantation of surgical clips and were prepared to receive radiotherapy. CT and MR images were acquired on the same day for each patient. Three radiation oncologists independently assigned cavity visualization score (CVS) and delineated the tumor bed based on first the CT then the MR images. Interobserver variability was assessed by volumes, generalized conformity index $\left(\mathrm{CI}_{\text {gen }}\right)$ and the distance between the centers of mass $(\mathrm{dCOM})$. Differences in mean values for parameters were tested by paired $t$-test or one-way analysis of variance, as appropriate.

Results: First, the mean volumes of tumor bed derived from MR were $22 \%, 27 \%$ and $21 \%$ smaller than those based on CT images for each observer. In addition, the mean $\mathrm{CI}_{\text {gen }}$ was significantly superior, and dCOM was smaller for MR than for CT images $\left(\mathrm{CI}_{\text {gen }}\right.$ : $0.59 \mathrm{vs}$ $0.52, P=0.008$; dCOM: $1.30 \mathrm{~cm}$ vs $1.39 \mathrm{~cm}, P=0.095$ ). Moreover, the mean CVS was 3.23 \pm 1.34 and $2.43 \pm 0.92$ for MR and CT images, respectively $(P=0.035)$. Last, a positive association was observed between the CVS and $\mathrm{CI}_{\text {gen }}$ for both modalities $(P<0.01)$.

Conclusion: Compared to CT, MR can improve the visualization of changes in the postoperative tumor bed. In addition, MR can yield a more precise definition of the tumor bed and improve the consistency of tumor bed contouring in patients without surgical clips.

Keywords: breast cancer, radiotherapy, magnetic resonance simulation, target volume delineation

\section{Introduction}

Breast cancer is the most common malignant disease in women worldwide, with an incidence of $24.5 \%$ of total cases in $2020 .{ }^{1}$ In China, there were approximately 268,600 new cases of breast cancer and 69,500 deaths attributed to breast cancer in 2015. ${ }^{2}$ Breast-conserving therapy is considered the standard of care for patients with early-stage breast cancer. ${ }^{3}$ As an indispensable part of breast-conserving therapy, adjuvant radiotherapy can reduce the local recurrence rate ${ }^{4,5}$ and improve the 15 -year overall survival rate. ${ }^{5}$ 
Precisely defining the tumor bed volume is critical in providing both a boost to the tumor bed and accelerated partial breast irradiation (APBI), which determines treatment effects and the dosage to organs at risk to some extent. However, it is commonly acknowledged that this key procedure is the weakest link in the breast-conserving radiotherapy process, which has some uncertainties and difficulties in clinical practice. Imaging modalities play an important role during radiotherapy, especially for simulation and target definition, leading to an accurate dose delivery to target volume. Computed tomography (CT), the standard and most common imaging modality in breast radiotherapy at present, has some limitations, such as limited soft tissue contrast and poor visualization of postsurgical changes. ${ }^{6-8}$ Therefore, it is difficult to precisely identify tumor bed based on CT images alone, and even experienced radiation oncologists tend to produce interobserver variability (IOV). ${ }^{6,7,9}$ Specifically, for patients without surgical clips as visible markers in the tumor beds, ${ }^{10}$ CT seems not to be a sufficient imaging tool for delineating the postoperative tumor bed. Compared with CT, magnetic resonance (MR) is better in visualizing soft tissue and distinguishing various boundaries and shapes of tumor bed from glandular tissue. ${ }^{11}$ In addition, MR imaging is a sensitive modality and has already commonly been used in detecting breast tumor at diagnosis and screening for recurrences during follow-up. ${ }^{12}$ As a consequence, MR imaging has potential applications for breast radiotherapy treatment planning.

MR simulators that are applicable for radiotherapy are rare around the globe, and there has been no report on the common use of MR simulation alone in breast radiotherapy. Recently, researchers have explored the role and feasibility of MR in breast radiotherapy, ${ }^{13,14}$ while an increasing number of studies have focused on comparing MR with CT simulation in defining and delineating the tumor bed for radiotherapy after breast-conserving surgery. Some of these groups believed that MR can provide clearer visualization of breast lumpectomy cavity and improve accuracy and consistency in delineating tumor bed. ${ }^{15-17}$ However, others found that there were no differences in target volumes or the IOV between two modalities. ${ }^{8,18,19}$ Most of previous studies were conducted among western populations, but there was inherent difference in breast density among various race or ethnicity. ${ }^{20,21}$ The variation possibly influenced the identification of tumor bed volumes and normal breast tissues and the present conclusions may also not be suitable for Chinese population, especially for those without surgical clips implanted during the breast-conserving surgery. Therefore, this study aims to compare the impact of CT and MR modalities on tumor bed delineation in breastconserving radiotherapy without the aid of surgical clips.

\section{Materials and Methods Study Population}

The study was approved by Institutional Review Board of Peking Union Medical College Hospital (approval No. ZS1643), and all participants signed written informed consents. The study population consisted of pathologically confirmed unilateral invasive breast carcinoma or mucinous carcinoma with clinical $\mathrm{T}_{1-2} \mathrm{~N}_{0} \mathrm{M}_{0}$ patients who had undergone ipsilateral breast-conserving surgery and prepared for adjuvant breast radiation therapy. Patients who had a history of malignant disease or breast radiotherapy, concurrent pregnancy or lactation, neoadjuvant treatment, or oncoplastic surgery or were not eligible for MR imaging were excluded.

\section{Simulation and Image Acquisition}

Both computed tomography (CT) and magnetic resonance (MR) simulation were performed in patients placed in a comfortable supine position with both arms above the head. Conventional immobilization device (a Med-Tec 250 breast board with a suitable upslope) or a special immobilization device (Styrofoam plus wedge plate) was used during CT simulation by one radiation therapist and one radiation oncologist. While for MR simulation, patients were immobilized by the special Styrofoam plus wedge plate with the same inclination angle as CT scans using body radiofrequency coils placed in the body radiofrequency coil bridge to prevent from breast deformation (Figure 1). ${ }^{22} \mathrm{CT}$ images were obtained using a CT simulator (Big Bore, Philips, Holland) with a 5-mm slice thickness. MR images were acquired with a $1.5 \mathrm{~T}$ simulator (Area, Siemens, Germany) with a 4-mm slice thickness. Lead wire for CT and cod-liver oil capsule for MR were placed on the surgical scars for those without periareolar incision. CT images and MR images, including T1weighted imaging (T1WI), T2-weighted imaging (T2WI) and diffusion-weighted imaging were acquired within one day for each patient (Table S1). The images were imported to Varian Eclipse planning system (Varian Medical Systems, Palo Alto, USA) for contouring. 


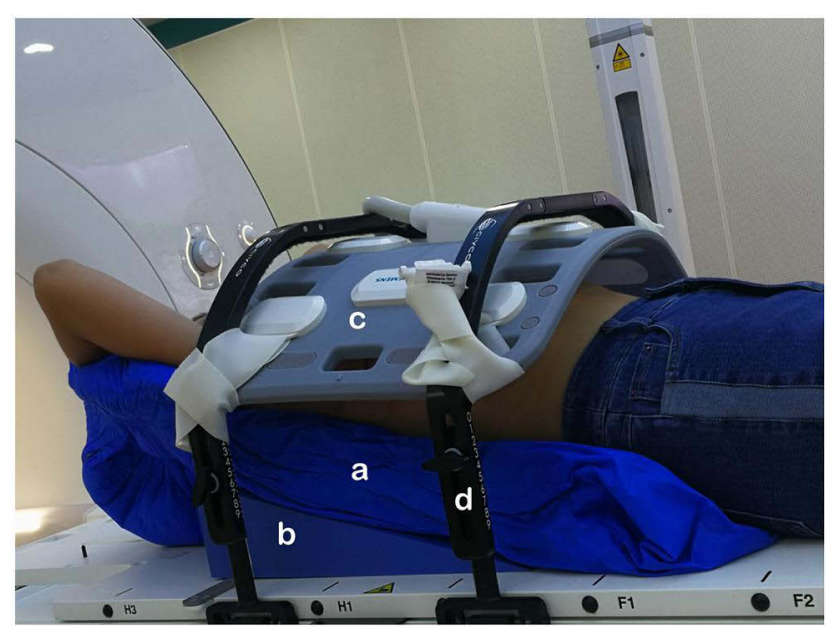

Figure I Magnetic resonance simulation body positioning system of immobilized patients. a, styrofoam; b, wedge plate; c, radiofrequency coils; d, adjustable bridges.

\section{Target Volume Delineation}

Three radiation oncologists participating in this study as observers independently delineated the tumor bed according to the following consensus guidelines. First, on the set of CT images, three observers assigned a cavity visualization score (CVS) to the tumor bed according to criteria proposed by Smitt et al and then delineated the tumor bed, including hematoma, seroma and any postoperative changes. ${ }^{23}$ Preoperative imaging including ultrasound or MR images, surgical records and postoperative pathology were provided when contouring. At least two weeks later, the observers assigned each MR image a CVS on a scale of 1 to 5. Then, they delineated the tumor bed on the T2WI fat saturation sequence with the support of other sequences and medical records. All the observers were blinded to the outlines delineated by the other two radiation oncologists as well as those of their own based on different modalities.

\section{Definition}

CVS values assessed by three observers on CT and MR were obtained for each patient. We calculated the mean CVS values of the three observers for the two modalities (presented as $\mathrm{CVS}_{\mathrm{CT}}$ and $\mathrm{CVS}_{\mathrm{MR}}$ ) in each patient. The difference between MR- and CT-evaluated mean CVS values by three observers was defined as CVS difference $\left(\mathrm{D}_{\mathrm{CVS}}\right)$, which was calculated as $\mathrm{CVS}_{\mathrm{MR}}$ minus $\mathrm{CVS}_{\mathrm{CT}}$ of the same patient. $A D_{C V S}>0$ indicates that the $\mathrm{CVS}_{\mathrm{MR}}$ is higher than the $\mathrm{CVS}_{\mathrm{CT}}$.

To compare the IOV between the CT and MR simulations, the following two indices were measured or captured. First, generalized conformity index $\left(\mathrm{CI}_{\text {gen }}\right)$ was defined as the sum of overlapping volumes of all possible pairs divided by the sum of unions of all possible pairs; ${ }^{24}$ a higher ratio indicated a smaller IOV. Second, the distance between the centers of mass (dCOM) was quantified by the distance between the centers of tumor bed of each observer pair, a lower ratio signaled a smaller IOV. In addition, $\mathrm{R}-\mathrm{CI}_{\text {gen }}$ of each patient was calculated as the ratio of the $\mathrm{CI}_{\text {gen }}$ from $\mathrm{MR}\left(\mathrm{CI}_{\text {gen, }} \mathrm{MR}\right)$ to the $\mathrm{CI}_{\text {gen }}$ from $\mathrm{CT}\left(\mathrm{CI}_{\text {gen, }}\right.$ СT). Consequently, a $\mathrm{R}-\mathrm{CI}_{\text {gen }}>1$ implies that $\mathrm{CI}_{\text {gen, } \mathrm{MR}}$ is higher than $\mathrm{CI}_{\text {gen, CT, }}$, and the magnitude of the value reflects the extent to which MR improves CTassessed $\mathrm{CI}_{\text {gen }}$. The definition of R-dCOM was similar to that of $\mathrm{R}-\mathrm{CI}_{\mathrm{gen}}$, as the ratio of the MR- to CT-evaluated $\mathrm{dCOM}$ for each patient. Therefore, a $\mathrm{R}-\mathrm{dCOM}<1$ means that the dCOM from MR is smaller than that from CT.

\section{Statistical Analysis}

Differences in the means between imaging modalities were assessed using paired $t$-test. One-way analysis of variance was used to compare tumor bed volumes among different observers on each modality. Student's $t$-test was performed to assess the difference between different subgroups evaluated by IOV parameters including $\mathrm{CI}_{\text {gen }}$ and $\mathrm{dCOM}$. The relationships between CVS value and IOV parameters were measured by Pearson correlation, and the Pearson correlation coefficient $(r)$ was calculated. All data were analyzed using SPSS (version 22.0), with two-sided significance defined as $P<0.05$.

\section{Results}

Between August 2018 and March 2019, a total of twenty patients with $\mathrm{T}_{1-2} \mathrm{~N}_{0} \mathrm{M}_{0}$ breast cancer were eligible for this study (Table S2); all of them underwent full-thickness closure without surgical clips. The median age of the enrolled patients was 48 years old (range, 33-73), and the median time interval from surgery to CT was 33.5 days (range, 23-50).

\section{Tumor Bed Volume}

Comparisons between the mean volumes of tumor bed on CT and MR modalities contoured by different observers are shown in Table 1. The mean reductions in the tumor bed volumes from $\mathrm{CT}$ to $\mathrm{MR}$ were $22 \%, 27 \%$ and $21 \%$ for observers 1, 2 and 3, respectively. The differences were highly significant (observers 1, 2 and 3: $P=0.001, P<0.001$ and $P=$ 0.011 , respectively). No significant difference was found in 
Table I Mean Volumes $\left(\mathrm{cm}^{3}\right)$ of the Tumor Bed Between CTand MR

\begin{tabular}{|l|c|c|c|c|c|}
\hline & \multicolumn{2}{|c|}{ CT } & \multicolumn{2}{c|}{ MR } & Inter Modality \\
\cline { 2 - 5 } & Mean & SD & Mean & SD & $P$ \\
\hline Observer I & 14.5 & 8.5 & 11.3 & 7.5 & 0.001 \\
\hline Observer 2 & 16.5 & 10.6 & 12.0 & 8.2 & $<0.001$ \\
\hline Observer 3 & 14.8 & 10.4 & 11.7 & 7.9 & 0.011 \\
\hline Inter observer $P$ & \multicolumn{2}{|c|}{0.589} & \multicolumn{2}{|c|}{0.834} & \\
\hline
\end{tabular}

Abbreviations: $\mathrm{CT}$, computed tomography; MR, magnetic resonance; SD, standard deviation.

the volumes of tumor bed delineated on $\mathrm{CT}$ alone $(P=0.589)$ or MR alone $(P=0.834)$ between the different observers.

\section{Cavity Visualization Score}

The mean \pm SD of the CVS was $2.43 \pm 0.92$ for $\mathrm{CT}$ and 3.23 \pm 1.34 for MR, with an obvious difference between the two modalities $(P=0.035)$. For fifteen of the patients, CT-based CVS values were improved by MR images. In addition, the mean CVS difference between MR and CT was $1.11 \pm 0.57$ with a maximum difference of 2.34. Among all the patients, only one had a lower CVS from MR in comparison with CT, and the other four cases had the same score for CT and MR.

\section{Interobserver Variability}

Measurements of consistency between observers for CTand MR-based definition of the tumor bed were evaluated by $\mathrm{CI}_{\text {gen }}$ and dCOM. $\mathrm{CI}_{\text {gen }}$ based on MR was significantly higher than that based on $\mathrm{CT}$ (means $\pm \mathrm{SD}, 0.59 \pm 0.13$ vs $0.52 \pm 0.09, P=0.008$ ), while $\mathrm{dCOM}$ was closer on MR than that on $\mathrm{CT}$, although the difference was not statistically significant (means $\pm \mathrm{SD}, 1.30 \pm 0.28$ vs $1.39 \pm 0.18 \mathrm{~cm}$, $P=0.095)$. Next, we divided patients into two groups according to the degree of $\mathrm{D}_{\mathrm{CVS}}$ : $\mathrm{D}_{\mathrm{CVS}}<1$ (CVS were not increased or not obviously increased by MR) and $\mathrm{D}_{\mathrm{CVS}}$ $\geq 1$ (CVS were obviously improved by MR in comparison with CT). There were 11 and 9 patients in the two groups, respectively. Statistically significant differences in $\mathrm{R}-\mathrm{CI}_{\text {gen }}$ $(1.24 \pm 0.20$ vs $1.04 \pm 0.15, P=0.018)$ and R-dCOM $(0.85$ \pm 0.17 vs $1.01 \pm 0.14, P=0.033)$ were observed between $\mathrm{D}_{\mathrm{CVS}} \geq 1$ and $\mathrm{D}_{\mathrm{CVS}}<1$ groups.

Four examples of tumor beds contoured by the three observers based on different imaging modalities are shown in Figure 2. Significant differences were observed in tumor beds delineated by the 3 observers as reflected by the different CVS values (high CVS for patient A, 5 and 4.7 for MR and CT, respectively; low CVS for patient B, 2 for both MR and CT). Consequently, poor visualization of tumor bed cavity and margins may lead to wide variations in the delineations defined by different observers. For patients $\mathrm{C}$ and $\mathrm{D}$, there were large differences in the CVS between MR and CT scans ( $\mathrm{D}_{\mathrm{CVS}}: 2$ and 2.4 for patients $\mathrm{C}$ and $\mathrm{D}$, respectively). Specifically, compared with MR images that could distinguish tumor bed from peripheral tissues, CT images only showed a slight change in density in the tumor bed with indistinct margins.

Figure 3 illustrates the relationship between the CVS and IOV parameters on MR and $\mathrm{CT}$ images for all patients. Independent of the imaging modality, there was a highly positive correlation between CVS and $\mathrm{CI}_{\text {gen }}(\mathrm{CT}: r=0.699$, $P=0.001$; MR: $r=0.895, P<0.001)$. The MR-assigned CVS
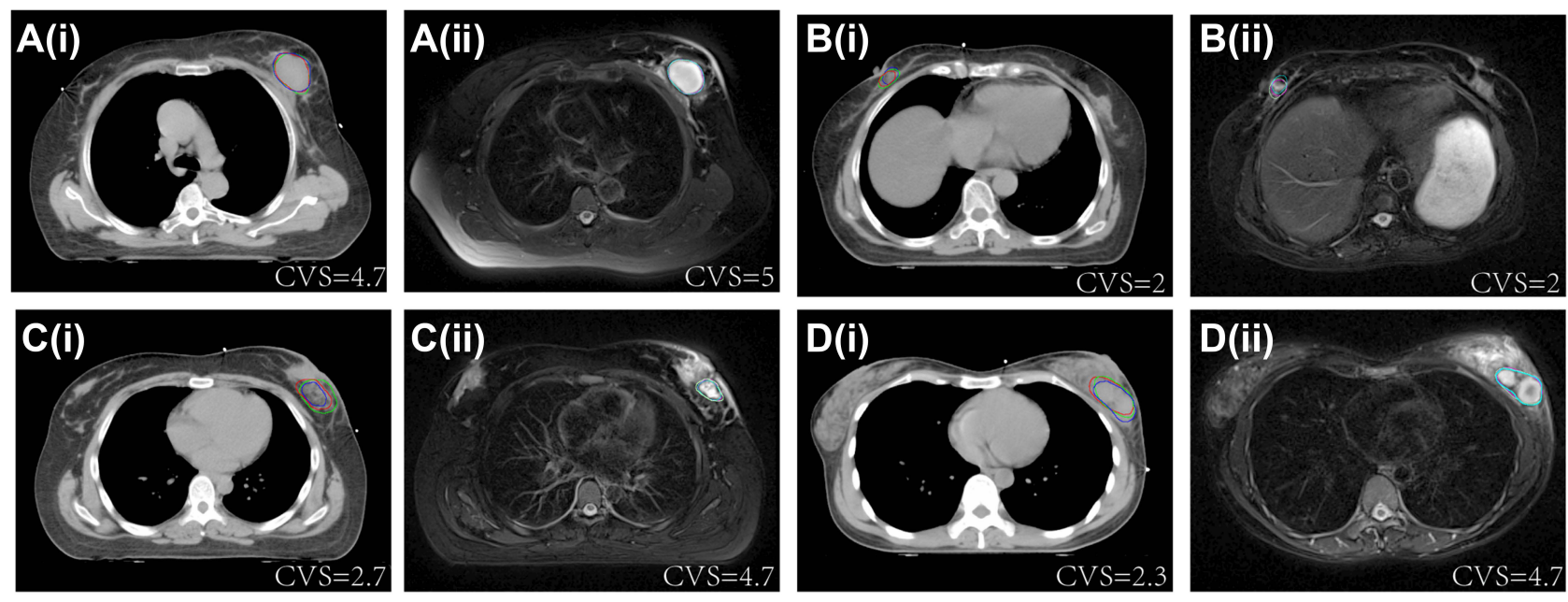

Figure 2 CT (i) and MR (ii) based delineations by three observers in four examples (A-D patients) with different cavity visualization scores. 

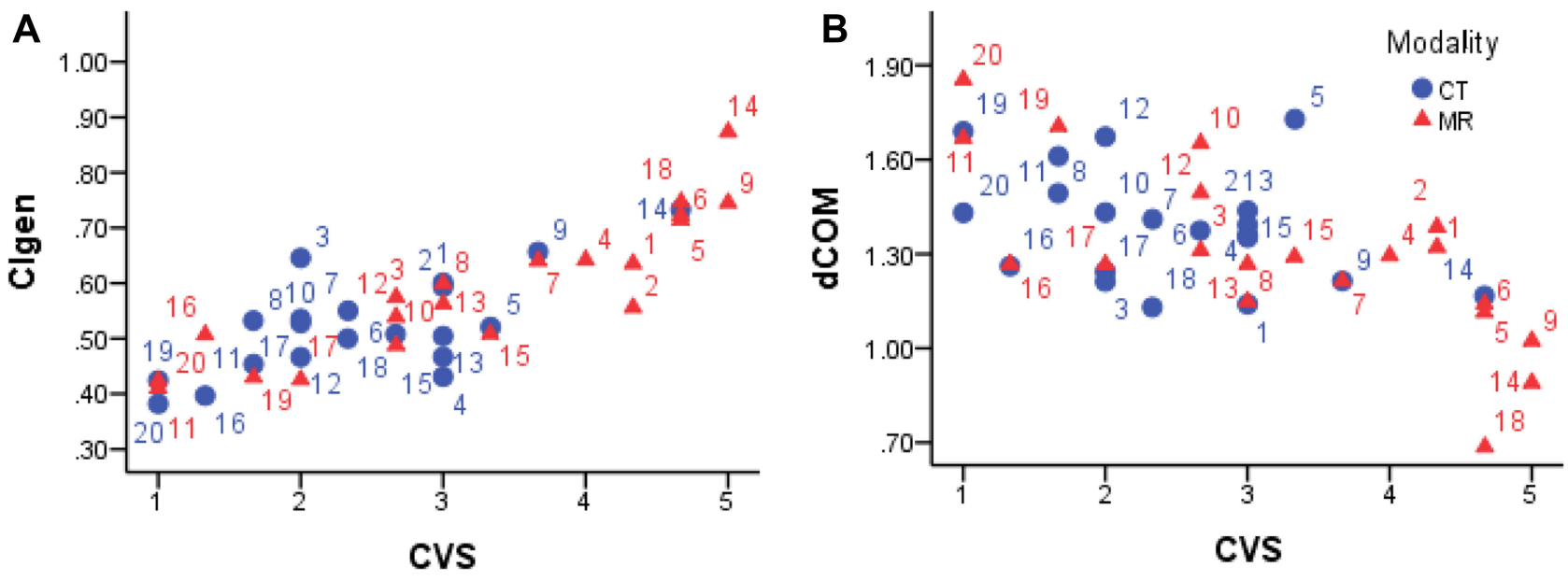

Figure 3 (A) Generalized conformity index (Clgen) and (B) the distance between the centers of mass (dCOM) as a function of cavity visualization score (CVS) for CT and MR based delineations of tumor bed. Patients' numbers are indicated for the two modalities, respectively.

was negatively associated with $\mathrm{dCOM}(r=-0.752, P<$ 0.001). For CT simulation, along with the increased CVS, dCOM showed a downward trend, but no statistical significance was found in this correlation $(P=0.125)$.

\section{Discussion}

In the present study, we investigated the potential advantages of MR simulation modality on the definition and delineation of postoperative tumor bed in patients undergoing breastconserving surgery without implantation of surgical clips.

As a result of reducing the risk of local recurrence, whole breast irradiation followed by tumor bed boost was recommended for most of patients after breast-conserving surgery. ${ }^{22,23}$ In addition, APBI, whose target volume only includes the tumor bed and adjacent breast tissue, has recently been proposed for some patients with low-risk early-stage breast cancer. $^{3,25}$ Regardless of the use of boost or APBI, tumor bed localization is critical but complex due to the variability and uncertainty, especially in instances without surgical clips. ${ }^{7,26}$ Traditionally, preoperative ultrasound or MR imaging, postoperative scars, and surgical clips or seroma on CT images were used to help delineate the tumor bed. However, there were challenges in accurately identifying and delineating tumor bed in these commonly used methods, which may lead to geometric miss of target volume and thereby increase the risk of local recurrence. ${ }^{6,27}$

Regarding tumor bed volumes, no significant differences were found in the tumor bed volumes delineated on CT images among the three observers in our study. However, using CT images alone, the locations and centers of tumor bed obviously varied among the different observers: in some patients with dense breast tissue, we found that the density of the tumor bed on CT was quite similar to that of surrounding glandular tissue. Therefore, radiation oncologists were unable to localize the precise boundary or shape of tumor bed without the assistance of surgical clips, which caused a low interobserver agreement of CT-based contouring. Similarly, previous studies demonstrated that CT may be unable to fastidiously distinguish breast tissue from fibrosis and has especially poor visualization in patients with dense breast tissue. ${ }^{6,7,27}$ Consequently, in clinical practice, for patients without surgical clips, radiation oncologists would expand margins of target volumes to prevent geometrical miss at the expense of an increased risk of radiation-related acute or late complications. Compared with CT, MR resulted in obviously smaller volumes of tumor bed from all the observers in the current study. CT-based tumor bed volumes may contain a small portion of normal breast tissue, which partly explains the reason for larger volumes delineated by CT images. Likewise, a previous report on patients without surgical clips implantation during surgery indicated that volumes assessed on MR were $30 \%$ to $40 \%$ smaller than those obtained from CT images. ${ }^{17}$ Additionally, research by Jolicoeur et al revealed significant differences in the average volumes delineated from the CT images between different observers, but no significant differences in the volumes assessed from the MR images were noted among the observers. ${ }^{17}$ However, in our study, there were no significant differences in tumor bed volumes derived from both CT and MR images among the three observers. This discrepancy may be due to different information offered to observers in the two studies. Observers in the present study were able to obtain preoperative imaging, surgical records and postoperative 
pathology as references when contouring; by contrast, the above information was unavailable in the other study, and the observers could only delineate based on the simulation images. This further increased the difficulty in delineation, and the differences in CT-based volumes by different observers were shown.

IOV was a potential factor affecting the accuracy in delineating tumor bed. Guidelines was reported to improve consistency of contouring in patients with breast cancer. ${ }^{25,26}$ Therefore, consensus guidelines were made before delineation as described above in our study. As parameters reflecting the $\mathrm{IOV}$, the $\mathrm{CI}_{\text {gen }}$ was significantly higher on $\mathrm{MR}$ images than on CT images $(P=0.008)$, while the dCOM was smaller on MR compared with $\mathrm{CT}$, but no statistical significance was observed $(P=0.095)$. Based on our results, MR has been shown to reduce the IOV and improve the consistency of tumor bed delineation. Differences in the IOV between the two modalities may be due to various degrees of visualization of tumor bed, and interobserver agreement of the tumor bed delineation depends directly on the visibility of cavity and margins. According to the statistical results, the CVS on the MR images was significantly higher than on the CT images $(P=0.035)$, and the use of MR improved the ability to visualize the tumor bed in $75 \%$ of the cases, resulting in better interobserver concordance and accuracy of MR images than CT images. In addition, we found that the interobserver consistency was significantly higher in patients with higher CVS values. It is easy to understand that observers can localize and delineate the tumor bed more accurately if they have better visualization of the cavity. On the other hand, for cases with a low CVS, it is difficult for even experienced radiation oncologists to determine the center location and boundary of tumor bed directly on images. Similarly, Mast et al reported that the mean conformity index values of lumpectomy cavity volumes were significantly higher for cases with a CVS $\geq 4$ compared with those with a CVS $<4$ for both CT and CT-MR fusion images. ${ }^{19}$ Although none of the patients included in the present study underwent adjuvant chemotherapy and the duration between surgery and imaging acquisition was less than 50 days. Seventeen of 20 cases were assessed as CVS $\leq 3$ on CT images, which meant that the margins of the tumor bed on CT images were indistinct or partly distinct for most cases. However, for some of these cases with unclear margins on CT, MR was able to visualize mostly distinct or clearly identified borders and distinguish small-volume seromas from normal tissue. This result conforms to the previously reported advantage of MR images in visualizing irregular seroma and differentiating postoperative changes and glandular breast tissue. ${ }^{16}$ More importantly, a significant difference in the IOV between the two different degrees of $\mathrm{D}_{\mathrm{CV}}$ was observed. The result indicates that the difference between CT- and MR-based IOV values is related to the difference in the visibility of the tumor bed between the two modalities.

Furthermore, we found that as CVS increased, interobserver consistency increased while dCOM showed a decreasing trend. Similar to our findings, a study comparing CT and MR images from 33 patients delineated by 11 observers, reported a negative correlation between the CVS and IOV parameters. ${ }^{28}$ Two previous studies also revealed a positive association between the CVS and $\mathrm{CI}_{\text {gen, }}{ }^{15,18}$ but neither reported a correlation coefficient or $P$ values as we have done in the current study. Moreover, MR was reported to improve CTassessed CVS in 11 cases (92\%) in another study, ${ }^{15}$ while the corresponding number in our study was 15 cases $(75 \%)$. These results were on par with those reported previously, which demonstrated that lumpectomy cavities were more visible in images of four different MR sequence images than in CT images. ${ }^{16}$ Despite the assistance of surgical clips, 14 of 15 patients $(93 \%)$ showed an increase in the CVS by at least 1 point when comparing MR with $\mathrm{CT}$, and the remaining 1 patient had the same CVS for both imaging modalities. ${ }^{16}$ By comparing the CVS derived from CT, MR-T1WI and MRT2WI, Jacobson and colleagues found that the differences between MR- and CT-based CVS were significant $(P<$ 0.001 ), and the T2WI showed superior visualization of the lumpectomy cavity. ${ }^{29}$ By contrast, the study by Giezen et al of 15 patients revealed that MR and CT presented a similar visibility of tumor bed, and the $\mathrm{CI}_{\text {gen }}$ of the MR images was lower than that of the CT images. ${ }^{18}$ One probable reason for these differences was that surgical clips were more visible on CT images, which may contribute to improvements in agreement with contouring. Even so, Giezen and colleagues suggested that MR highlighted more details of the interfaces of lumpectomy cavity in cases with a high CVS. ${ }^{18}$ Moreover, another study of 10 patients and 4 observers found no significant differences in lumpectomy cavity volumes and the IOV between CT and CT-MR coregistered images. ${ }^{19}$ Differences in imaging modalities and target volume delineation procedures may contribute to the disparities. In the present study, observers independently delineated the tumor bed first on CT images and then on MR images after at least two weeks, and the other one was not displayed when delineating on a modality. In a study by Mast et al, the CT images were first delineated, and then modifications were made on the CT-MR fusion images. ${ }^{19}$ This nonblinded approach may introduce bias; therefore, no significant differences in the tumor bed volume or IOV were 
observed between the CT and CT-MR images in their study. Unfortunately, for patients with a low CVS on MR images, MR cannot improve the interobserver consistency. For these patients, there was no significant difference in the tumor bed delineations between the two modalities in this study. MR simulation, with its high cost and long acquisition time, was reported to take an additional 16 minutes to obtain T1WI and T2WI sequences when compared to the duration of a CT scan in a previous study. ${ }^{11}$ It took us approximately 23 minutes to complete the entire scan of MR simulation. Therefore, largescale clinical application of MR simulation faces difficulties with limited benefits in some cases.

We acknowledge that there are several limitations in the present study. First, the observers in our study may not be as familiar with delineating the tumor bed on MR images as on CT images despite the existence of consensus guidelines to reduce bias. Second, the number of patients and observers is limited, and the large-scale application of MR faces difficulties. Therefore, further study with a larger cohort is necessary to identify factors that affect the visibility of the tumor bed to screen patients who will likely benefit from MR simulation in the breast radiotherapy treatment planning.

\section{Conclusions}

This study showed that MR can improve the visualization of the tumor bed in comparison with CT images to some extent, resulting in improvements in the interobserver consistency of tumor bed delineations in patients without surgical clips implanted in tumor bed. However, for patients with low cavity visualization scores on MR images, MR is unable to improve interobserver concordance and accuracy of tumor bed contouring.

\section{Abbreviations}

APBI, Accelerated partial breast irradiation; $\mathrm{CI}_{\text {gen, }}$ Generalized conformity index; CT, Computed tomography; CVS, Cavity visualization score; dCOM, The distance between the centers of mass; IOV, Interobserver variability; MR, Magnetic resonance; T1WI, T1-weighted imaging; T2WI, T2-weighted imaging.

\section{Ethical Approval}

The study protocol was approved by the Institutional Review Board of Peking Union Medical College Hospital (approval No. ZS-1643), and all participants signed written informed consents. All procedures performed in studies involving human participants were in accordance with the ethical standards of the institutional and/or national research committee and with the 1964 Helsinki declaration and its later amendments or comparable ethical standards.

\section{Acknowledgments}

The authors are grateful to all staff members from the Department of Radiation Oncology, Peking Union Medical Hospital for their contributions in the preparation of this manuscript.

\section{Funding}

This study was supported by Ministry of Science and Technology of the People's Republic of China (No. 2016YFC0105207); and the College Student Research and Innovation Project of Beijing (No. 2018zlgc0636).

\section{Disclosure}

The authors declare that they have no conflicts of interests.

\section{References}

1. Sung H, Ferlay J, Siegel RL, et al. Global cancer statistics 2020: GLOBOCAN estimates of incidence and mortality worldwide for 36 cancers in 185 countries. CA Cancer J Clin. 2021;71(3):209-249. doi: $10.3322 /$ caac. 21660

2. Chen W, Zheng R, Baade PD, et al. Cancer statistics in China, 2015. CA Cancer J Clin. 2016;66(2):115-132. doi:10.3322/caac.21338

3. Darby S, McGale P, Correa C, et al. Effect of radiotherapy after breast-conserving surgery on 10-year recurrence and 15-year breast cancer death: meta-analysis of individual patient data for 10,801 women in 17 randomised trials. Lancet. 2011;378 (9804):1707-1716. doi:10.1016/S0140-6736(11)61629-2

4. Bartelink H, Maingon P, Poortmans P, et al. Whole-breast irradiation with or without a boost for patients treated with breast-conserving surgery for early breast cancer: 20-year follow-up of a randomised Phase 3 trial. Lancet Oncol. 2015;16(1):47-56. doi:10.1016/S14702045(14)71156-8

5. Clarke M, Collins R, Darby S, et al. Effects of radiotherapy and of differences in the extent of surgery for early breast cancer on local recurrence and 15-year survival: an overview of the randomised trials. Lancet. 2005;366(9503):2087-2106. doi:10.1016/S01406736(05)67887-7

6. Landis DM, Luo W, Song J, et al. Variability among breast radiation oncologists in delineation of the postsurgical lumpectomy cavity. Int J Radiat Oncol Biol Phys. 2007;67(5):1299-1308. doi:10.1016/j. ijrobp.2006.11.026

7. Petersen RP, Truong PT, Kader HA, et al. Target volume delineation for partial breast radiotherapy planning: clinical characteristics associated with low interobserver concordance. Int J Radiat Oncol Biol Phys. 2007;69(1):41-48. doi:10.1016/j.ijrobp.2007.01.070

8. Kirby AM, Yarnold JR, Evans PM, et al. Tumor bed delineation for partial breast and breast boost radiotherapy planned in the prone position: what does MRI add to X-ray CT localization of titanium clips placed in the excision cavity wall? Int J Radiat Oncol Biol Phys. 2009;74(4):1276-1282. doi:10.1016/j.ijrobp.2009.02.028

9. Hurkmans C, Admiraal M, van der Sangen M, Dijkmans I. Significance of breast boost volume changes during radiotherapy in relation to current clinical interobserver variations. Radiother Oncol. 2009;90(1):60-65. doi:10.1016/j.radonc.2007.12.001 
10. van Mourik AM, Elkhuizen PH, Minkema D, Duppen JC, van Vlietvroegindeweij $\mathrm{C}$. Multiinstitutional study on target volume delineation variation in breast radiotherapy in the presence of guidelines. Radiother Oncol. 2010;94(3):286-291. doi:10.1016/j.radonc.2010.01.009

11. Whipp EC, Halliwell M. Magnetic resonance imaging appearances in the postoperative breast: the clinical target volume-tumor and its relationship to the chest wall. Int $J$ Radiat Oncol Biol Phys. 2008;72(1):49-57. doi:10.1016/j.ijrobp.2007.12.021

12. Clauser P, Mann R, Athanasiou A, et al. A survey by the European Society of Breast Imaging on the utilisation of breast MRI in clinical practice. Eur Radiol. 2018;28(5):1909-1918. doi:10.1007/s00330017-5121-4

13. van Heijst TC, den Hartogh MD, Lagendijk JJ, van den Bongard HJ, van Asselen B. MR-guided breast radiotherapy: feasibility and magnetic-field impact on skin dose. Phys Med Biol. 2013;58 (17):5917-5930. doi:10.1088/0031-9155/58/17/5917

14. Metcalfe P, Liney GP, Holloway L, et al. The potential for an enhanced role for MRI in radiation-therapy treatment planning. Technol Cancer Res Treat. 2013;12(5):429-446. doi:10.7785/ tcrt.2012.500342

15. Al-Hammadi N, Caparrotti P, Divakar S, et al. MRI reduces variation of contouring for boost clinical target volume in breast cancer patients without surgical clips in the tumour bed. Radiol Oncol. 2017;51(2):160-168. doi:10.1515/raon-2017-0014

16. Huang W, Currey A, Chen X, et al. A comparison of lumpectomy cavity delineations between use of magnetic resonance imaging and computed tomography acquired with patient in prone position for radiation therapy planning of breast cancer. Int J Radiat Oncol Biol Phys. 2016;94(4):832-840. doi:10.1016/j.ijrobp.2015.12.014

17. Jolicoeur M, Racine ML, Trop I, et al. Localization of the surgical bed using supine magnetic resonance and computed tomography scan fusion for planification of breast interstitial brachytherapy. Radiother Oncol. 2011;100(3):480-484. doi:10.1016/j.radonc.2011.08.024

18. Giezen M, Kouwenhoven E, Scholten AN, et al. MRI- versus CT-based volume delineation of lumpectomy cavity in supine position in breast-conserving therapy: an exploratory study. Int J Radiat Oncol Biol Phys. 2012;82(4):1332-1340. doi:10.1016/j.ijrobp.2011.05.008

19. Mast M, Coerkamp E, Heijenbrok M, et al. Target volume delineation in breast conserving radiotherapy: are co-registered $\mathrm{CT}$ and $\mathrm{MR}$ images of added value? Radiol Oncol. 2014;9:65. doi:10.1186/ 1748-717X-9-65
20. El-Bastawissi AY, White E, Mandelson MT, Taplin S. Variation in mammographic breast density by race. Ann Epidemiol. 2001;11 (4):257-263. doi:10.1016/s1047-2797(00)00225-8

21. Del Carmen MG, Halpern EF, Kopans DB, et al. Mammographic breast density and race. AJR Am J Roentgenol. 2007;188 (4):1147-1150. doi:10.2214/AJR.06.0619

22. Paulson ES, Erickson B, Schultz C, Allen LX. Comprehensive MRI simulation methodology using a dedicated MRI scanner in radiation oncology for external beam radiation treatment planning. Med Phys. 2015;42(1):28-39. doi:10.1118/1.4896096

23. Smitt MC, Birdwell RL, Goffinet DR. Breast electron boost planning: comparison of CT and US. 10.1148/radiology.219.1.r01ap34203. Radiology. 2001;219(1):203-206. doi:10.1148/radiology.219.1. r01ap34203

24. Kouwenhoven E, Giezen M, Struikmans H. Measuring the similarity of target volume delineations independent of the number of observers. Phys Med Biol. 2009;54(9):2863-2873. doi:10.1088/ 0031-9155/54/9/018

25. Theberge V, Whelan T, Shaitelman SF, Vicini FA. Altered fractionation: rationale and justification for whole and partial breast hypofractionated radiotherapy. Semin Radiat Oncol. 2011;21(1):55-65. doi:10.1016/j.semradonc.2010.08.007

26. Corrao G, Rojas DP, Ciardo D, et al. Intra- and inter-observer variability in breast tumour bed contouring and the controversial role of surgical clips. Med Oncol. 2019;36(6):51. doi:10.1007/s12032-019-1273-1

27. Hepel JT, Evans SB, Hiatt JR, et al. Planning the breast boost: comparison of three techniques and evolution of tumor bed during treatment. Int J Radiat Oncol Biol Phys. 2009;74(2):458-463. doi:10.1016/j.ijrobp.2008.08.051

28. Pogson EM, Delaney GP, Ahern V, et al. Comparison of magnetic resonance imaging and computed tomography for breast target volume delineation in prone and supine positions. Int J Radiat Oncol Biol Phys. 2016;96(4):905-912. doi:10.1016/j.ijrobp.2016.08.002

29. Jacobson G, Zamba G, Betts V, Muruganandham M, Buechler-Price J. Image-based treatment planning of the post-lumpectomy breast utilizing CT and 3TMRI. Int J Breast Cancer. 2011;2011:246265. doi: $10.4061 / 2011 / 246265$

\section{Publish your work in this journal}

Cancer Management and Research is an international, peer-reviewed open access journal focusing on cancer research and the optimal use of preventative and integrated treatment interventions to achieve improved outcomes, enhanced survival and quality of life for the cancer patient.
The manuscript management system is completely online and includes a very quick and fair peer-review system, which is all easy to use. Visit http://www.dovepress.com/testimonials.php to read real quotes from published authors. 